\title{
Data Realities in Plural Contexts: Appraisal of a Definition [of Social Informatics]
}

\section{Fletcher Cole}

School of Information Systems, Technology \& Management, University of New South Wales, Sydney 2052 f.cole@unsw.edu.au

The meaning of "context" within the definition of social informatics (SI) is explored. The most general context of interest is formed by the relationship between the broad categories of the "technological" and the "social', as they are manifest in information and communication technologies (ICTs). Within and between these broad categories there are numerous distinct but inter-related contexts. Contextual complexity at a more detailed level is illustrated through close examination of the data-related references in a textual description of geographic information systems (GIS). A six-category taxonomy is used to highlight the complex cross-referencing between inter-related vocabularies that reflects the multiple contextual realities involved. They reveal themselves as open to multiple and continuing interpretation within different contexts at different levels of conceptualisation.

Practical implications include suggestions that current generalist approaches to systems design may be too removed from the specifics of the task at hand, and that information professionals in their education and training need to be exposed to a wide range of perspectives, reflecting the various human contexts in which their technical expertise is likely to be deployed. What is desirable is a sophisticated appreciation of the many contexts in which ICTs function.

Introduction

The aim of this paper is to illustrate and explore the meaning of "context" within the definition of social informatics (SI). The intersection of a number of contexts seems to characterize social informatics at a general level, but it also seems to be a feature of the detailed practice of information professionals. A practical outcome of this exploration might be a closer integration of the social and the technological in professional education, as well as a more systematic theoretical account of their relationship.

The term "context" was originally associated with the interpretation of written texts, where it was seen as the text surrounding a particular text, which significantly contributed to understanding its 
meaning. This surrounding text is now sometimes referred to as "co-text" (Lyons 1995: 271). The original definition was extended in anthropology by Bronislaw Malinowski $(1923,1935)$, with his ideas of "context of situation" and "context of culture", to take into account the wider social and cultural environment of a text (or utterance) and, subsequently, the entire range of human activity (Goodwin \& Duranti 1992).

Context is a key idea in the definition of social informatics under view, which is that proposed by Rob Kling: "Social Informatics refers to the interdisciplinary study of the design, uses, and consequences of ICTs that takes into account their interaction with institutional and cultural contexts" (Kling et al, 2005: 6).

The focus of this definition is a particular set of information and communication technologies (ICTs), and a relationship of "interaction" with their "institutional and cultural" or "larger social context" (Sawyer \& Eschenfelder 2002: 428, 433). The context that initially attracts attention is that defined by social, cultural and technological dimensions. As a guiding definition the terms used are necessarily broad and therefore open to widely varying interpretation. To proceed systematically beyond this preliminary representation some of the complexity and analytical alternatives need to be identified and elaborated.

First, the general relationship between the social and the technological will be discussed. Then, at a more detailed level, a specific example will be analysed. In this example we find the idea of data in various working definitions and usages in a descriptive text about geographic information systems (GIS). What becomes apparent is that the meaning of "data" varies in complex ways. The concept-in-use is not one, but many, which take(s) into account a number of contextual fields, a number of realities perhaps, as a matter of course.

\section{Defining Context at Large: Social \& Technological}

The basic claim motivating the initial definition is that "ICTs do not exist in social or technological isolation" (Kling et al, 2005: 6). ICTs are seen as not existing in "isolation", but within a wider "context", the "social", and the "cultural and institutional". They also exist within the context of the "technological". To some this is unremarkable and obvious, and yet it is not so clear that these relationships are taken much into account in the deployment of ICTs, or in our various policy, managerial, educational or academic descriptions of them (Kling et al 2005).

There are differing viewpoints on the link between these areas, with different understandings of "context" as a consequence. The relationship between the social (or its nominalised institutional manifestation as 'society') and the technical or the technological ('technology' ) is often couched in terms of some dynamic (MacKenzie \& Wajcman 1999). In the social informatics definition here the dynamic is viewed as "interaction", a term which itself is open to a variety of interpretations.

In order to put this issue in its own context, it is useful to sketch several basic alternative interpretations of the relationship between society and technology. These alternatives frame, at 
the most general level, the various ideas of context that might be adopted within social informatics.

\section{ICTs in social context (Technology in Society)}

From this viewpoint technology is distinct from society; it is autonomous and independent. Society employs ICTs for social purposes (among other things). The dynamics of the relationship is that technology influences society. The "interaction" consists of "pro-action" (on the part of technology) and "re-action" (often "resistance" on the part of society) (Habermas 1987).

Overcoming "barriers" to implementation is a prime concern for those who view such a relationship optimistically. Functionalist and instrumentalist approaches to ICT design usually adopt this as their theoretical frame, when they care to make it explicit (Hirschheim 1995: 69-73). A more symmetrical position is that society provides the environment for ICTs. Technology is distinct from society, and influences society, but society also influences the very design of technology (Sawyer \& Eschenfelder 2002).

\section{ICTs as part of social context (technology partially social)}

A more complex understanding of technology is adopted when ICTs are seen to exhibit social aspects, whose significance become important when deployed in society, but which nevertheless remain subordinate to the technological. The notion of "interaction" is complicated by the blurring of the distinction between society and technology (MacKenzie \& Wajcman 1999). Socio-technical studies can usually be located here (Rogers 1997).

\section{ICTs have technological and social contexts (technology and society)}

ICTs exhibit social and technological aspects, all of which are integral to their constitution. Any technology (such as ICTs) is both technological and social. The technological aspects can be distinguished from the social, and these give a technology its distinctive cultural characteristics. There is no technology which does not exhibit social characteristics; these are seen to be foundational and inescapable. Therefore characterizing the relationship between the between the social and the technological as "interaction" is somewhat problematic: "interwoveness" is probably a more precise metaphor from this point of view (Bowker et al 1997, Heath \& Luff 2000, Schuurman 1980).

\section{ICTs are primarily social (technology as social)}

ICTs are constructed by human communities adopting a certain view of their situation ("reality"); "technology" is a socially-constructed assemblage of material practices (and that's enough) 
(Haraway 1997; Law 2003). In principle, these practices have no greater human significance than other human activities (as cross-cultural anthropological comparisons would suggest). As with all human notions, technological ideas exert a certain power over us, and so should be subject to critical evaluation. Any "interaction" which takes place with and in "society" relates to the deployment of "discipline" and distribution of "power", sometimes invoking ideas of "emancipation" and the like (Foucault 1977; Habermas, 1984).

This sketch of alternative viewpoints suggests that the terms "social" and "technological" themselves have ranges of meaning that need clarification. A broad and multi-faceted view of the social is implied in the social informatics definition. Anything which is manifest in "Society" (in general) and in social institutions is relevant (e.g. politics, economics, language, ethics, art, music, religion). Here "social" means "cultural" (in a social anthropological sense). From this point of view it is logical to see technology as an element of the cultural.

Others adopt a more analytically rigorous approach and distinguish the social from other aspects of human life and activity. Entire disciplinary and practice communities have formed on the basis of such distinctions. However, such rigour does not necessarily help much in comprehending the relationship with technology, and may in fact be taken as grounds for discounting it.

Similarly, technology is a multi-faceted notion, which in most literatures is surprisingly vague in definition, perhaps because of its numerous cultural manifestations. Distinguishing the facets of technology can be achieved in several ways; first of all, along disciplinary lines, represented by disciplines such as computer science, chemical engineering, and telecommunications (for instance).

However, such an analytical frame is not at all straightforward, as technologies usually draw on combinations and overlaps of disciplinary traditions, including those in supporting and foundational roles (e.g. mathematics). Technology can also be defined according to their "application domains" or, expressing this more generally, the areas of human endeavour in which they are manifest, including society at large (e.g. management information systems, medicine, naval architecture, environmental science).

Social informatics defines its subject in terms of a widely adopted label for a cluster of technologies that are manifest in society, viz. ICTs. The domain is necessarily ill-specified, with very open borders. The central idea hovers around notions of "artifacts and practices" associated with "information" and "communication". It may have topics in such diverse areas as software testing, systems design and indexing (Kling et al, 2005: 11). The intention is to invite many different approaches to the subject matter, especially those arising from inter-disciplinary concerns. Given the previous discussion, inter-disciplinary approaches are inevitable, both in specific studies and in social informatics in general.

These various notions of social and technical have implications for how context is understood. 
Each discipline, application domain, with their associated practice communities form contexts of their own. So also do the various overlaps and combinations of these, and specific institutional settings represent distinct contexts also. Depending on the point of view adopted, each of these contexts might be seen to exhibit aspects of the social, and the technological, whether viewed in broad (comprehensive and undifferentiated) or narrow (differentiated and specific) terms.

For instance, Sawyer \& Eschenfelder (2002), in their review of SI, seem to cover both bases, first defining social context (broadly) as "a holistic sense of interaction among levels of social analysis, and the particular characteristics that help to define any given level of analysis" or organizational unit of analysis (2002: 435, 453). Secondly, they align their definition more closely with an Actor Network Theory view of context as "socio-technical networks of influences" (453), so that ideally social informatics "investigates how the influencers and nodes in a socio-technical network shape each other" (453).

In elucidating the nature of context in terms of possible technological, social and cultural relationships we need to proceed with care. We need to be aware of the range of meanings, whether these are made explicit or not in any particular study. This plurality is not only evident at the most comprehensive levels of social informatics, it is also visible in the detailed ways various contexts are shaped and renewed in the interaction between the social and the technological. The next section provides an instructive example of the multiplicities involved.

\section{Defining Context in Detail: Data Realities in GIS}

Our example concerns the references to "data" within the context of geographic information systems (GIS). Data is very mundane idea, fundamental to disciplinary knowledge. It is also a major prerequisite of any information system. Whether computer-related or not, an information system needs data to digest. So it is hardly surprising that, as the use of computers has spread, various ideas about data have emerged in practice, almost to the point of anarchy (Machlup 1983: 646-649). What counts as "data" implicates and is implicated by the particular settings, organizational, social and systems settings, to which the data variously belong. Interpreting "data" inescapably involves understanding "context'. To systematically identify these various contextually-related usages is a challenge.

GIS examples are often interesting in the way they handle a wide range of complex spatial and temporal data. This is evident in the wide discussion of fundamental data issues in the considerable body of theoretical and practical literature about GIS (Longley, 1999; Sheppard 2001). The example reported here involves a short extract from a standard textbook concerning GIS. The purpose of highlighting (or bracketing) the text differs from that of the text itself, but rather is to examine how the data-related vocabulary is used. The text follows.

Current GIS are most successful at handling static, easily identifiable units in geographical space, and data structuring and handling of more complex data, particularly with a temporal component, continues to challenge GIS developers. In the last few years, data-base research 
has been dominated by developments in the object-oriented approach and whilst this approach has benefited the structuring of entity data (especially of easily defined objects, such as property parcels, and utility infrastructure, and offers possibilities for spatio-temporal data handling), there are difficulties in applying it to continuous field data where permanent relations do not exist.

(Burrough \& McDonnell 1998: 294)

One of the features of this passage is the way the terminology related to data originates from several distinct disciplinary sources. Reading these data terms correctly involves identifying and understanding their respective disciplinary contexts. Other data-related texts exhibit a much narrower range of reference. However, because of its multi-disciplinary provenance, GIS description often has call to refer to several disciplines - viz. our sample. In order to explicate this text in more detail, some general observations on data-related vocabularies and the construction of a means of analysis are needed first.

\section{Preliminary Remarks: Data in General}

The basic problem to be solved by computer-based information systems, in their current manifestations, as far as data is concerned, is to reconcile two broad ways in which data is understood (Molenaar 1998:7; Suchman \& Trigg 1993: 172; Worboys 1995: 3). The first of these belongs to the realm of computer design, in which data is rendered manipulable ("computable"). The second belongs to the work, organizational and social contexts to which the data is relevant (the "application domain", from a computer science and information systems point of view). On closer examination these two realms appear within themselves far from simple, and the relationship between them is profound. In particular, there seem to be a number of distinct and complex ways of characterizing data.

A casual examination of academic and technical literature quickly leads to the observation that "data" is defined differently by different disciplinary or practice communities. In the GIS literature, where our sample text originates, the terminology of a number of disciplines is evident. A hydrographer is concerned with data of "elevation", "slope", drain direction", "location", "volume", etc. A programmer works with (ANSI-defined) data types, such as "integer", "char", "floating point", etc. GIS experts, in their own right, seek to represent "geography" in terms of "points", "lines", "polygons", and "rasters", and the rest. Complex data structures, built out of simpler elements, are referred to by different terminology again ("BLOBs", "bags" and "arrays") And the use of specialized synonyms ("text", "strings") and homonyms ("object", "type") serve to confuse further (c.f. standard texts such as Burrough \& McDonnell 1998; Elmasri \& Navathe 2004).

In order to cluster these terminologies and put them into some systematic order related to their origins in the two data realms of organizational setting and computer design, a classification scheme has been devised, as a preliminary instrument, consciously provisional in character (Cole 2005). It sets out various categories of data-term usage in order to separate the apparent 
differences in vocabulary. This scheme was used to analyse the terminology in the sample text. What is important to note in the list that follows is that each category defines a different and distinctive context for the terms that belong there. The data-term categories are:

1. Setting: data-related terms here designate the specific named organizational, business, or social location or workplace in which they are used, including settings such as a department, sub-unit, classroom, consulting room, etc., and in relation to particular named institutions. It is here that the significance of any data and the functioning of any system is finally defined for its users.

2. Semantic / Theme: terms here designate the general subject domains, or disciplinary topics and themes. They identify the semantic rather than the syntactic properties of the data (e.g. hydrology, human demography, finance, etc.) From a systems point of view they, along with the first category, are the prime target of application.

3. Dimension / Task / Function: terms here characterize the data for the task at hand, and are often defined according to some dimension or general function (time, space) rather than a semantic quality. The field / object distinction in GIS circles seems to work at this level (Cova \& Goodchild, 2002).

4. Structure: the terms in this category identify data complexes, made up of formalized clusters of more primitive data (next category). Where a computer-related system is involved the terms seem to fall into two major groups - one to do with the complexity of the data being represented (e.g. user-defined data types), and another group related to the efficient design of computer storage (file structure) (Worboys \& Duckham 2004; Elmasri \& Navathe 2004).

5. Primitive: These are "atomic" data types or data "primitives", from a computer scientist's point of view (integer, date, char, etc.) - although their precise definitions have their own complexities (MacKenzie 1996). "Type" is not used to label this category, because it so often assumes other meanings in other categories.

6. Medium: This category relates not to how data is represented, but how it is inscribed in some medium, even if ephemeral. Terms related to the representation of file structures in a computer are regarded as complex data (category 4). Detecting the possibility of data in signal patterns or media inscriptions is the basis for several disciplines, as wide ranging as cryptography and physical bibliography.

0. Undifferentiated: This further category proved necessary when it came to using the taxonomy to analyze texts. It includes vague terms, which are insufficiently clear to be categorized, or which seem to refer to many categories simultaneously (e.g. "data set").

Further examples in their categories are provided in Cole (2005). No claim is made that the scheme and its category labels are universally applicable, heavily skewed as they are to computer and systems-related situations. Similar broad categorizations, but not as refined, have been suggested elsewhere, especially for geographic systems, such as distinguishing the "semantic" 
from the "technical" in Burrough \& McDonnell (1998: 294), or closer to the scheme outlined here, the identification of levels of data abstraction in Rhind \& Green (1988) and Peuquet (1984).

\section{Analysis of the GIS Text}

The classification just outlined was put to its intended use, that of differentiating actual data-vocabularies-in-use in an examination of the sample text. The adopted procedure was to re-format the text to isolate direct or indirect references to data. Each reference was then labelled with the data category to which it was seen to belong. This resulted in the following annotations:

\begin{tabular}{|c|c|}
\hline Text, with Data-Related Terms Highlighted & Data Category \\
\hline Current GIS are most successful at handling static, easily identifiable units & [3. dimension? \\
\hline in geographical space, & [2. semantic \\
\hline and data structuring and handling of more complex data, & $\begin{array}{l}\text { [0. undifferentiated?[3. } \\
\text { dimension? }\end{array}$ \\
\hline particularly with a temporal component, & [3. dimension \\
\hline \multicolumn{2}{|l|}{$\begin{array}{l}\text { continues to challenge GIS developers. In the last few years, data-base } \\
\text { research has been dominated by developments in }\end{array}$} \\
\hline the object-oriented approach & [4. structure \\
\hline $\begin{array}{l}\text { and whilst this approach has benefited the structuring of entity data } \\
\text { (especially of easily defined objects, }\end{array}$ & [3 dimension \\
\hline such as property parcels, and utility infrastructure, & [2. semantic \\
\hline and offers possibilities for spatio-temporal data handling), & [3. dimension \\
\hline $\begin{array}{l}\text { there are difficulties in applying it to continuous field data where permanent } \\
\text { relations do not exist. }\end{array}$ & [3. dimension \\
\hline
\end{tabular}

As noted previously, in this particular text the idea of "data" is manifest in several distinct vocabularies, each defining a particular context. Most evident is that a number of data categories are referred to within a comparatively short span, and that there is cross-referencing among them. Not all texts are like this, of course. More typical is a data-related lexicon of a more circumscribed context, that of a single discipline (e.g. software design, geography). Other texts relate to nearly the full range of categories (Cole 2005).

Here the majority of references are to terms in three of the six categories: [2. semantic, [3. dimension, and [4. structure. The terms in these categories seem to be critical in enabling the successful translation between the different contexts in which data is represented. Also noted is the frequent cross-referencing between terms of different categories. Terms may qualify terms in other categories, to varying degrees: e.g. the [3. dimension term "static, easily identifiable units" is further qualified by a [2. semantic reference to "geographical space".

Terms can be extensive and complex phrasal expressions. These can be self-defining, as in the 
example: "continuous field data where permanent relations do not exist". To follow this through in some detail here: "where permanent relations do not exist" explains "continuous field data". The "permanent relations" being referred to are those of [3. dimension, so identifying the category of data to which both phrases belong. This rules out other implications, such as "continuous field data" being a reference to the context of [4. structure, for then the explanation would probably be in terms of the tessellation of a field, for instance. Nor is it a reference to utilizing data as [5. primitives, for then an explanation is likely to refer to pixels. Identifying the context is part and parcel of comprehending the text.

Cross-referencing between categories can be uncertain however. For instance, the phrase "more complex data" is a vague catch-all, i.e. [0. undifferentiated, but alternatively could be regarded as referring to [3. dimension, given the qualifying phrase "particularly with a temporal component". However, not completely, because "particularly with" indicates the possibility other forms of "complexity", not necessarily in this category (a software engineer could read it as a term of [4. structure). No help is provided by the reference to "data structuring", used here with a vague, general meaning, not specifically related to aspects of [4. structure as defined in this taxonomy. Context here is characterised by ambiguity.

Plurality of meaning is particularly evident in the way structural, dimensional and semantic references to "data" are tightly woven together in the word "object". In the second sentence of the extract, the "object-oriented approach" to data, (a reference to a closely-defined software term of [4. structure) is said to provide a "beneficial" structural base for data conceptualized as "entities" and "easily defined objects" (synonyms referring to [3. dimension) These are then are given further meaning by references at a [2. semantic level, as "property" data, etc. It helps the reader to know something of the 'technical' context of the terms.

\section{Conclusions about Data}

Data is not a primitive invariant concept. As illustrated by our sample text, its meaning may vary in complex ways, and be derived from the specific disciplinary and practical contexts in which it is used. The preponderance of terms from [2. semantic, [3. dimension, and [4. structure categories, and the cross-referencing between them, appear to facilitate the overall process of representing "data in organization" within the pragmatics of "computer system'.

The translation from the one broad domain to the other appears to take place in smaller two-way moves, or sequences of moves, and it is these moves which are indexed by the different data-vocabularies we have observed. The process is not smooth however. Incommensurate terms from different categories are juxtaposed, in no discernable sequence - and it is up to readers to unravel it, to make the connections and make sense of it all. The working rule is that "what is being said in one text-unit is assumed to be relevant to what is being said in the immediately preceeding text-units" (Lyons 1995: 264). This may not work for all readers, and we are only given a certain amount of help. 
A sign of readers" knowledgeability will be the ease with which they sort out the conceptual cross-referencing (the indexicals), and their ability to resort to assemblages of accepted precedents and conventions. It is an opportunity for them to demonstrate their competence at utilising contextual clues as specialist readers (Livingston 1995). The text cannot be understood in isolation, divorced from its context, nor isolated from the activity of reading it.

From a slightly different point of view, the meaning of "data" can be seen to arise out of the association or interaction of a number of different disciplinary contexts. The process also creates additional specific relational contexts through the complex cross-referencing that we have observed. It may be a distortion, therefore, with practical consequences, to attend to the meaning of "data" at a single contextual level, without acknowledging, at least tacitly, the possibility of a

wider context and range of reference. This multiple deployment contributes to its overall meaning and creates its own context. It creates a broad context of meaning, data-within-GIS, represented by the text as a whole, and announced in our text at the outset: "Current GIS are ..." .

\section{Conclusions about Context}

Contexts which are salient for social informatics are represented, in part, by the "interaction" between several disciplinary and workplace communities that give rise to descriptions such as our sample text. A sweeping reference to "context" may be useful as a preliminary, but further thought is required to identify broader and narrower contexts, and ones created out of their inter-relationships. Our analysis often needs more explicit specification of relevant contexts, even though they might seem "self-evident".

Some conclusions from our discussion might be:

1. Contexts are created by the definition and continual re-definition of a discipline (or area of practice) and its several sub-disciplines, as well as in its documentation and associated literature.

2. Contexts are also created by the cross-referencing between disciplines, or meaning-levels (as in our sample text). The context resides in their relationship.

3. Contexts are created by clusterings of other contexts, incorporating disciplinary and relational contexts $(1 \& 2)$, as illustrated by our text in its totality, but represented as well by massive social and cultural contexts (such as those referred to in our definitions of SI). This means that fundamental concepts (such as 'data' in GIS) in their very detail are likely to assume complex sets of contextually-defined meanings.

4. An aspect of all contexts will be Malinowski's original point about identifying the relationship of texts to the rest of life: to other documentation, to conversation, to regular and routine practice. This will be closely associated with some sort of social or cultural collective effort (Douglas 1992: 240), or membership of some "community" (Van Maanen \& Barley 1984), a "community-of-practice" perhaps, (Lave \& Wenger 1991). 


\section{Practical Implications}

By dint of necessity practitioners generally find they have to invoke social as well as technological dimensions in order to accomplish the work they do (Law, 2003: 3). However this is not necessarily reflected in the emphases in our professional training and education. New recruits need to be exposed to a wide range of perspectives reflecting the various human contexts in which their technical expertise will be deployed.

Although the complex references to "data" might be recognized implicitly in day-to-day practice, there is also a practical advantage in acknowledging its complexities more explicitly, so that we move away from habits of mind that think of it as "primitive". For instance, the current generalist approaches to systems design, even though social aspects are increasingly being taken into account, still may be too removed from the translation tasks that need to be undertaken when faced with specifics, especially the specifics of data-in-context. While there may be similarities between socio-technical systems, it is the differences, the contingencies, the required translations and transcriptions that seem to matter more for workability.

An inclusive attitude towards the human is not always reflected in what is deemed to be technically relevant in, say, organizational policy documentation regarding our information systems and data management. We might need to more expansive in our recognition of social and organizational dimensions in the framing of inter-organizational procedures, such as cataloguing rules and data re-use standards.

One of the aspirations of social informatics is to be multi-disciplinary, and accommodating of a wide variety of studies. It calls for analytical concepts that incorporate both the "social" and the "technological" in balanced measure. Presumptions about the relationship between these broad cultural contexts give rise to different interpretations of that relationship, but as well they open up numerous opportunities to explore the multiplicity of contexts within and between them.

\section{References}

Bowker, G.C., Star, S.L., Turner, W. \& Gasser, L. (eds.) (1997) Social Science, Technical Systems, and Cooperative Work Mahwah:.Lawrence Erlbaum Associates

Burrough, P.A. \& McDonnell, R.A. (1998) Principles of Geographical Information Systems 2nd ed. Oxford: Oxford University Press

Cole, F. (2005) The discourse of data: Exploring data-related vocabularies in geographic information systems description Journal of Information Science 31(1), 44-56

Cova, T.J. \& Goodchild, M.F. (2002) Extending geographical representation to include fields of spatial objects International Journal of Geographical Information Science 16(6), 509-532

Douglas, M. (1992) Rightness of categories In Douglas, M., \& Hull, D. (eds.) How Classification Works: Nelson Goodman among the Social Scientists 239-271. Edinburgh: Edinburgh University Press 
Elmasri, R. \& Navathe, S.B. (2004) Fundamentals of Database Systems 4th ed. Reading: Addison-Wesley

Foucault, M. (1977) Discipline and Punish: The Birth of the Prison Harmondsworth: Penguin

Goodwin, C. \& Duranti, A. (1992) Rethinking context: An introduction In Duranti, A. \& Goodwin, C. (eds.) Rethinking Context: Language as an Interactive Phenomenon 1-42. Cambridge: Cambridge University Press

Habermas, J. (1984) The Theory of Communicative Action Boston: Beacon Press

Habermas, J.; Shapiro, J.J. (trans.) (1987) Toward a Rational Society: Student Protest, Science, and Politics Cambridge: Polity

Haraway, D. (1997) Modest_Witness@Second_Millenium London: Routledge

Heath, C. \& Luff, P. (2000) Technology in Action Cambridge: Cambridge University Press

Hirschheim, R.A., Klein, H.K. \& Lyytinen, K.J. (1995) Information Systems Development and Data Modelling: Conceptual and Philosophical Foundations Cambridge: Cambridge University Press

Kling, R., Rosenbaum, H. \& Sawyer, S. (2005) Understanding and Communicating Social Informatics: A Framework for Studying and Teaching the Human Contexts of Information and Communication Technologies Medford, NJ: Information Today

Lave, J. and Wenger, E. (1991) Situated Learning: Legitimate Peripheral

Participation Cambridge: Cambridge University Press

Law, J. (2003) Networks, Relations, Cyborgs: on the Social Study of

Technology http://www.comp.lancs.ac.uk/sociology/papers/Law-Networks-Relations-Cyborgs.pdf(Centre for Science Studies, Lancaster University, Lancaster LA1 4YN, UK. Last revised on 6th December 2003; previously published 2000. )

Livingston, E. (1995) An Anthropology of Reading Bloomington: Indiana University Press

Longley, P.A. et al (eds.) (1999) Geographical Information Systems New York: Wiley

Lyons, J. (1995) Linguistic Semantics Cambridge: Cambridge University Press

Machlup, F. (1983) Semantic quirks in the study of information In Machlup, F. \& Mansfield, U. (eds.) The Study of Information: Interdisciplinary Messages 641-671 New York: Wiley

MacKenzie, D. (1996) Negotiating arithmetic, constructing proof In Knowing Machines: Essays on Technical Change 165-183. Cambridge MA: MIT Press

MacKenzie, D. \& Wajcman, J. (eds.) (1999) The Social Shaping of Technology 2nd ed.

Buckingham: Open University Press

Malinowski, B. (1923) The problem of meaning in primitive languages In Ogden, C.K. \& 
Richards, I.A. (eds.) The Meaning of Meaning 296-336. London: Kegan Paul

Malinowski, B. (1935) Coral Gardens and their Magic London: Allen \& Unwin

Molenaar, M. (1998) An Introduction to the Theory of Spatial Object Modelling for GIS London: Taylor \& Francis

Peuquet, D.J. (1984) conceptual framework and comparison of spatial data models Cartographica 21(4), 66-113

Rhind, D.W. \& Green, N.P.A. (1988) Design of a geographical information system for a heterogenous scientific community International Journal of Geographical Information Systems 2(2), 171-189

Rogers, Y. (1997) Reconfiguring the social scientist In Bowker, G.C. et al (eds.) Social Science, Technical Systems, and Cooperative Work 57-77. Mahwah: Lawrence Erlbaum Associates

Sawyer, S. \& Eschenfelder, K.R. (2002) Social informatics: Perspectives, examples, and trends ARIST: Annual Review of Information Science and Technology 36, 427-465

Schuurman, E.; Morton, H.D. (trans.) (1980) Technology and the Future: A Philosophical Challenge [Techniek en toekomst] Wedge, Toronto

Sheppard, E. (2001) Geographic information systems: Critical approaches In International Encyclopedia of the Social and Behavioral Sciences 9, 6182-6185 Amsterdam: Elsevier

Suchman, L.A. \& Trigg, R.H. (1993) Artificial intelligence as craftwork In Chaiklin, S. \& Lave, J. (eds.) Understanding Practice: Perspectives on Activity and Context 144-178. Cambridge: Cambridge University Press

Van Maanen, J. \& Barley, S.R. (1984) Occupational communities: Culture and control in organizations Research in Organizational Behavior 6, 287-365

Worboys, M.F. (1995) GIS: A Computing Perspective London; Taylor \& Francis

Worboys, M.F. \& Duckham, M. (2004) GIS: A Computing Perspective 2nd ed. Boca Raton: CRC Press 